Dedicated to Professor Norman March on the occasion of his 90th birthday.

\title{
Application of the plane-wave-based perturbation theory to the density modulation induced by a point charge in an electron gas
}

\author{
I. $\mathrm{Nagy}^{1,2}$ and M. L. Glasser ${ }^{3,4}$ \\ ${ }^{1}$ Department of Theoretical Physics, Institute of Physics, \\ Budapest University of Technology and Economics, \\ H-1521 Budapest, Hungary \\ ${ }^{2}$ Donostia International Physics Center, P. Manuel de Lardizabal 4, \\ E-20018 San Sebastián, Spain \\ ${ }^{3}$ Department of Physics, Clarkson University, Potsdam, \\ New York 13699-5820, USA \\ ${ }^{4}$ Departamento de Física Teórica, Atómica y Óptica, Universidad de Valladolid, \\ E-47071 Valladolid, Spain
}

(Dated: February 9, 2017)

\begin{abstract}
The induced electron density at the position of a single point charge $Z$ embedded in a threedimensional degenerate electron gas is studied at high densities. The perturbative, plane-wavebased treatment developed within the framework of idempotent density matrices by March and Murray [Phys. Rev. 120, 830 (1960)] is applied here up to second order in $Z$. Comparison with the result obtained by considering the exact scattering enhancement in a bare Coulomb field is made. The small numerical difference found in the second-order term of the induced density at contact is analyzed following Wigner's [Phys. Rev. 94, 77 (1954)] similar perturbative treatment of the proton field in the hydrogen atom. The impact of the many-body screening and nonidempotency of the host system on the perturbative results found are discussed as well.
\end{abstract}

PACS numbers: 02.30.Mv, 03.65.Nk, 03.75.Sc 


\section{INTRODUCTION}

The effect of charged impurities on the properties of a metal is of considerable physical interest, both because of the possibility of deliberately introducing them so as to study the physics of electron-atom interaction in metals, and because most real materials contain impurities which affect their physical properties. By using modern experimental techniques, such as STM and STS, one is able to consider in detail the modulations in the local density of states at the Fermi level. One can probe the induced density in the many-electron system of metals via positron annihilation or Knight-shift measurements with muons. Furthermore, one might consider in calculating the life-time of an added electron in a cold electron gas, the screening of the electron-electron interaction, needed to avoid the divergence in the electron, or intruder charge, self-energy close to the Fermi energy.

There are several instances in which simple approximation methods yield correct results even though the conditions for the applicability of those methods are not fulfilled. The best known example is the calculation of the Rutherford cross section in three dimensions by firstorder Born approximation, i.e., by using plane-wave states. Apart from this case, however, one should check the adequacy of this familiar approximation in potential scattering. Such a check is, surprisingly, particularly important in the case of short-range forces [1].

In the present work as a first step the electron gas is assumed to be noninteracting, but perturbed by the potential of an embedded point charge. Thus, the problem is one of quantum mechanical scattering of a single electron by the potential of an external charge. The thermodynamics then follows by filling up the new set of energy levels according to FermiDirac statistics corresponding to an ideal momentum distribution function, i.e., with unit occupation numbers for one-electron states up to the invariant Fermi level. The continuous spectra of the electron gas Hamiltonian and the perturbed Hamiltonian coincide.

The method applied rests on the well-known paper by March and Murray [2] where the idempotent density matrices, Dirac and canonical, were discussed in reference to imperfections (central fields) in metals. Concretely, we apply March and Murray's second-order prescription, Eq.(6.5) of their paper, to calculate the total electron charge at the position

of the embedded point charge in a high-density electron gas. Notice that the corresponding low-density limit was treated earlier [3] by us using their Eq.(4.12), i.e., a third-order differential equation, with a Hulthen-type potential. Since in the present paper we use plane-wave 
states and find a close similarity with the second-order term based on the exact Coulomb enhancement of scattered waves, we are tempted to refer for analogy to Wigner's secondorder perturbational calculation of the energy of the hydrogen atom. Remarkably, in order to provide a physical interpretation, Wigner pointed out that the finite result for the binding energy in his second-order treatment is due to the form $\left(\sim r^{-1}\right)$ of the Coulomb potential $[4]$.

\section{THEORY AND RESULTS}

According to Eq.(6.5) of March and Murray [2], the second-order perturbation expression for the total density $n(r=0)=n_{0}+n_{1}(r=0)+n_{2}(r=0)$ at the position of a charge $Z$ embedded in a paramagnetic electron gas at zero temperature is

$$
\begin{gathered}
n_{0}=\frac{1}{\pi^{2}} \int_{0}^{k_{F}} d k k^{2}=\frac{k_{F}^{3}}{3 \pi^{2}} \\
n_{1}(r=0)=\frac{2}{\pi^{2}} \int_{0}^{k_{F}} d k k \int_{0}^{\infty} d s V(s) \sin (2 k s) \\
n_{2}(r=0)=\frac{4}{\pi^{2}} \int_{0}^{k_{F}} d k \int_{0}^{\infty} d s V(s) \sin (2 k s) \int_{s}^{\infty} d t V(t) \sin (2 k t),
\end{gathered}
$$

where the ideal momentum distribution function $f_{0}\left(0 \leq k \leq k_{F}\right)=1$ for occupied oneelectron momentum eigenstates in a homogeneous degenerate system has been applied. $V(r)$ is the spherical central potential due to the point charge. In writing these equations the expression $x^{2} j_{0}(x) \bar{n}_{0}(x)=(1 / 2) \sin (2 x)$ is used for the product of the spherical Bessel $\left(j_{l}\right)$ and Neumann $\left(\bar{n}_{l}\right)$ functions..

Now, as in Wigner's perturbation theory for binding with an $1 / r$ potential, we consider first the unscreened case in our problem, i.e., we take $V(r)=Z / r$. From Eq.(2) we get

$$
n_{1}(r=0)=Z \frac{k_{F}^{2}}{2 \pi}
$$

In dealing with the second-order term in Eq.(3), we change the order of integration and introduce the new variable $t=x s$. Thus, we have

$$
n_{2}(r=0)=Z^{2} \frac{4}{\pi^{2}} \int_{0}^{k_{F}} d k \int_{1}^{\infty} d x \frac{1}{x} \int_{0}^{\infty} d s \frac{\sin (2 k s) \sin (2 k s x)}{s} .
$$

The integral with respect to $s$ becomes $I(x)=(1 / 2) \ln [(x+1) /(x-1)]$ independently of $k$. 
To perform the $x$-integration in Eq.(3), we employ a convergent $(x \geq 1)$ expansion

$$
I(x)=\sum_{m=0}^{\infty} \frac{1}{(2 m+1) x^{2 m+1}}
$$

by which the remaining integrations are elementary and finally we get

$$
n_{2}(r=0)=Z^{2} \frac{4}{\pi^{2}} k_{F} \sum_{m=0}^{\infty} \frac{1}{(2 m+1)^{2}}=Z^{2} \frac{k_{F}}{2}
$$

since the sum becomes $\lambda(2)=(3 / 4) \zeta(2)=\pi^{2} / 8$ in terms of Riemann's zeta-function.

Next, we turn to the exact treatment of scattering states. With Coulomb potential $Z / r$, the important enhancement factor $E_{C}(\eta)$ is

$$
E_{C}(\eta)=\frac{2 \pi \eta}{1-e^{-2 \pi \eta}} \equiv 1+\pi \eta+2 \sum_{m=1}^{\infty} \frac{\eta^{2}}{m^{2}+\eta^{2}}
$$

in terms of the Sommerfeld parameter $\eta=Z / k$. From this we get

$$
\begin{gathered}
n(0)=\frac{1}{\pi^{2}} \int_{0}^{k_{F}} d k k^{2} E_{C}(k)=n_{0}+Z \frac{k_{F}^{2}}{2 \pi}+Z^{2} \frac{2}{\pi^{2}} \sum_{m=1}^{\infty} \frac{1}{m^{2}}\left[k_{F}-\frac{Z}{m} \arctan \left(\frac{m k_{F}}{Z}\right)\right] \\
=n_{0}+\frac{Z}{2 \pi} k_{F}^{2}+\frac{Z^{2}}{3} k_{F}-\frac{Z^{3}}{\pi} \zeta(3)+\frac{\pi^{2} Z^{5}}{45 k_{F}}+O\left(k_{F}^{-3}\right) .
\end{gathered}
$$

In the high-density limit, where $k_{F} \gg 1$, this expression tends to

$$
n(0)=n_{0}+\frac{Z k_{F}^{2}}{2 \pi}+Z^{2} \frac{k_{F}}{3}-\frac{Z^{3}}{\pi} \zeta(3)
$$

where we used $\zeta(2)=\pi^{2} / 6$. Apart from its sign, the last term corresponds $[5,6]$ to the total density of the entire spectrum of bound states of a hydrogen-like atom. This $k_{F}$-independent term is negligible when $k_{F} \gg 1$.

By comparing the exact Eq.(10) with the perturbative Eq.(7), one can see a moderate numerical deviation in the $Z^{2}$-order term. This approximate agreement is one of the main results of this work. We can say, following Wigner's early observation, that the secondorder perturbation theory developed by March and Murray for charged imperfections in a noninteracting, degenerate electron gas appears to be meaningful.

In the rest of this note, we turn to important subquestions on the role of screening and non-idempotency of the host system. We focus on changes in the first-order term $n_{1}(0)$. By substituting into Eq. (2) a Yukawa-type potential $V_{Y}(r)=(Z / r) \exp (-\Lambda r)$ we get

$$
n_{1}^{(Y)}(r=0)=\frac{Z}{\pi^{2}}\left[\left(k_{F}^{2}+\frac{\Lambda^{2}}{4}\right) \arctan \frac{2 k_{F}}{\Lambda}-\frac{\Lambda k_{F}}{2}\right]
$$


which reproduces Eq.(4) when $\Lambda=0$. However, this transparent closed expression allows a deeper analysis of the $k_{F}$-dependence of the parameter $\Lambda\left(k_{F}\right)$. One can see that with conventional, Thomas-Fermi scaling of $\Lambda^{2}=4 k_{F} / \pi$ the high density limiting value, $Z k_{F}^{2} / 2 \pi$ will not change. In other words, at that limit the screened potential is penetrable for a very fast electron. One could reduce the numerical factor $(1 / 2 \pi)$ of the limiting form only with an $\Lambda \propto k_{F}$-scaling. In this manner the high-density pair-correlation function at contact, $g(0)=1-\left|n_{1}(0) / n_{0}\right|$ becomes tunable. Indeed, there are theoretical arguments $[7,8]$ that such scaling is the only one if the goal is to reproduce the exactly known asymptotic form for this function $g(0)=1-\left(1.4 / k_{F}\right)$.

We afinally come to the challenging problem of the non-idempotency encoded in the reduced one-particle density matrix in momentum space. The diagonal of this matrix is the one-particle momentum distribution function. Until now, we have used the ideal momentum distribution function $f_{0}\left(0 \leq k \leq k_{F}\right)=1$. Due to the correlated electron motion, the momentum distribution function $f(k)$ describing the population of plane-wave states of real particles (and not Landau's quasiparticles) in the translationally invariant system differs from the ideal momentum distribution function [9]. This is a crucial point since, for instance, in a mean-field Kohn-Sham-like treatments of Density Functional Theory with auxiliary orbitals the population of particles is still the ideal one. Therefore by that method one can not calculate the exact kinetic energy. In fact, all relative-coordinate-dependent many-body complications are hidden in an effective external field.

In order to appreciate the important role of non-idempotency $(N)$ in our problem, we take a simple [10] parametrized $\left(x \equiv k / k_{F}\right)$ expression from the literature

$$
\begin{aligned}
& f(0 \leq x \leq 1)=a(1)\left[1-a(2) x^{2}\right] \\
& f(x>1)=a(3) e^{-a(4)(x-1)}+\frac{T}{x^{8}}
\end{aligned}
$$

Here $a(4)=4, a(3)=(32 / 13) \delta, \delta=(1 / 3)-a(1)[(1 / 3)-(1 / 5) a(2)]-T / 5, a(2)=0.048 / k_{F}$, $a(1)=1-0.019 / k_{F}$, and $T=\left[2 /\left(3 \pi k_{F}\right)\right]^{2} g(0)$ in terms of the pair-correlation at contact. By performing the integration in Eq.(2) with a $Z / r$ potential, in leading order we get

$$
n_{1}^{(N)}(r=0) \cong \frac{Z k_{F}^{2}}{2 \pi}\left[1-\frac{0.042}{k_{F}}+O\left(1 / k_{F}^{2}\right)\right]
$$

As expected, $n_{1}^{(N)}(0)<n_{1}(0)$ due to the nonideality of the momentum distribution function. Relativistic effects $[11,12]$ which appear at extreme $k_{F}$, are beyond the scope of this note. 


\section{SUMMARY}

The induced electron density at the position of a single point charge $Z$ embedded in a three-dimensional degenerate electron gas is studied at high densities. The perturbative, plane-wave-based treatment developed within the framework of idempotent density matrices by March and Murray [Phys. Rev. 120, 830 (1960)] is applied here up to second order in $Z$. Comparison with the result obtained by considering the exact scattering enhancement in a bare Coulomb field is made. The small numerical difference found in the second-order term of the induced density at contact is analyzed following Wigner's [Phys. Rev. 94, 77 (1954)] similar perturbative treatment of the proton potential in the hydrogen atom.

The impact of many-body screening and non-idempotence in the host system on the perturbative results found are considered as well. Specifically, the impact of a non-ideal momentum distribution function could influence the two-term asymptotic results for the induced density at contact in cases with $V(r)= \pm(1 / r)$, i.e., with protons and antiprotons. However, Thomas-Fermi screening modifies the results obtained with bare interactions in a stronger manner. Clearly, further research on the combined effects of these ingredients needed for a physically self-consistent model are still desirable to proceed along the path marked out by the pioneering paper of March on charged imperfections.

\section{Acknowledgments}

This note is dedicated to Professor Norman March. The authors are indebted to him for many useful, enlightening discussions in the past, and hope that the future will allow further fruitful collaborations with him. One of us (MLG) acknowledges the financial support of MINECO (Project MTM2014-57129-C2-1-P) and Junta de Castilla y Leon (VA057U16)). 
[1] R. Peierls, Surprises in Theoretical Physics, (Princeton University Press, Princeton, 1979).

[2] N. H. March and A. M. Murray, Phys. Rev. 120, 830 (1960).

[3] I. Nagy, M. L. Glasser, and N. H. March, Phys. Lett. A 373, 3182 (2009).

[4] E. P. Wigner, Phys. Rev. 94, 77 (1954).

[5] O. J. Heilman and E. H. Lieb, Phys. Rev. A 52, 3628 (1995).

[6] N. H. March, I. A. Howard, I. Nagy, and P. M. Echenique, J. Math. Phys. 46, 072104 (2005).

[7] I. Nagy, J. I. Juaristi, R. D. Muino, and P. M. Echenique, Phys. Rev. B 67, 073102 (2003).

[8] M. Corona, P. Gori-Giorgi, and J. P. Perdew, Phys. Rev. B 69, 045108 (2004).

[9] A. A. Abrikosov, L. P. Gorkov, and I. E. Dzyaloshinski, Methods of Quantum Field Theory in Statistical Physics (Dover, New York, 1963).

[10] B. Barbiellini and A. Bansil, J. Phys. Chem. Solids 62, 2181 (2001).

[11] S. A. Chin, Ann. Phys. (New York) 108, 301 (1977).

[12] I. Nagy, J. Phys. B: At. Mol. Phys. 19, L421 (1986). 